\title{
Enhancing Engineering Education via Physical Experiments: The Case of Learning Energy Storage with a Flywheel System
}

\author{
Chung-Neng HUANG \\ National University of Tainan, Tainan, Taiwan \\ kosono@mail.nutn.edu.tw \\ Chen-Min CHENG \\ National University of Tainan, Tainan, Taiwan \\ noreg0083415@gmail.com \\ Jenn-Kun KUO \\ National University of Tainan, Tainan, Taiwan \\ jkkuo@mail.nutn.edu.tw
}

\begin{abstract}
Design thinking is complex that is hard to learn and harder to teach. The purpose of engineering education is to graduate engineers who can design (Dym CL, 2005). How to enhance students' reasoning and cognizing about data has gained an increasingly visible role in the K-12 curriculum (Doerr H. M. and Lyn D., 2003). In order to enhance teaching and learning engineering mathematics, a modeling approach by shifting the focus on finding solutions to a particular problem to create a system is proposed in this study. Besides, a training process for the college students who are intending to be the instructors of guiding physical experiments is included. Nonlinear systems, signifying any system is not linear, cannot satisfy the superposition principle. In this paper, a new modeling method for nonlinear systems based on an Adaptive Network-Fuzzy Inference System (ANFIS) for operation and practice was used (Jang, J.S.R., 1993). Through this method, students were able to learn how the transfer functions work for identifying an unknown system. This method had well helped the participated students how the nature of nonlinear system is and how to find out the optimal solutions in controls.
\end{abstract}

Keywords: engineering enhancing education (EEE), physical experiment, system modeling, electromagnetic flywheel (EF), Adaptive Network-Fuzzy Inference System (ANFIS).

\section{INTRODUCTION}

There are many types of training methods which can guide students to learn and distinguish what the linear and nonlinear systems are. Here, nonlinear regression is learned 
to be the common method for nonlinear modeling. This method is not only concise but also remains in the shape of mathematical model. However, it may increase more complicated nonlinear model which is difficult to be used in real applications. In order to avoid above problems and simplify the computation process in nonlinear modeling, the methods such as neural network, adaptive neural fuzzy systems etc. are always adopted. Although these methods can accurately fit the nonlinear system, for they are model-free methods which are difficult to let students observe the mathematical properties existing in these systems for further applications. For the educational target at EEE, instead of those complicated system modeling kits this study adopted and integrated the Taguchi method (Ho, W.H., et al., 2009) and ANFIS (Huang, C.N. and Chang, C.C., 2011) to achieve system identification works.

Nowadays, the energy issues have become one of the most important topics in the world. In order to shorten the distance between EEE and those most concerned issues in the world, an energy storage device, the electromagnetic flywheel (EF) system, was selected as the demonstration platform for EEE in this study. EF systems, with the progressing of high-strength and light-weight composite material, and technologies of power electronics, battery, bearings and controls, instead of traditional battery systems, become an alternative for energy storage issues. EF systems with a lot of complex nonlinear uncertainties, such as electromechanical coupling, magnetizing, and demagnetizing etc. that was selected to be the experiment platform to enhance the impression of what nonlinear system is and how the EF works for this EEE. For the EF system is characterized with lots of nonlinearity and uncertainties, there would be a lot of restrictions and difficulties existing in traditional mathematic ways for modeling. In this study, initially an EF system was design and set up, then through the proposed learning process to confirm the effectiveness of EEE. The works of the EF system in design, setup, and experiment etc. were accomplished by the participators, including advisor, students and the offerors of system parts.

\section{SYSTEM DESIGN \& SETUP}

According to the experimental demand for data collection, the EF system platform was designed by advisor and machine manufacturer. Where, the main components were marked as follows: (1) Motor / generator, (2) EF (in Fig. 2), (3) Switching box for charging and discharging control, (4) Power analyzer ( No. DM2436AB ), (5) Motor driver (DELTA $220 \mathrm{~V} / 2.2 \mathrm{~kW}$ ), (6) Control box of excitation voltage and current adjustment, (7) Control box for Manually loading. According to above design, the EF system was accomplished. Fig. 2 shows a motor/generator device coupled with a flywheel. Through this setup, the nature of energy transformation between kinetic and electric powers can be observed and recorded for analzing the nonlinearity. Table 1 shows the geometric size and parameters of EF system.

In order to let students understand the charging and discharging characteristics of the EF system, a series of required approaches for experiment were designed. In the approaches, nine sections in accordance with the frequency setups as 20, 25, 30, 35, 40, 45, 50, 55 and $60 \mathrm{~Hz}$ were given to observe and record the input/output energy data corresponding to 
different rotational speeds. In this experiment, the concerning data was automatically recorded from a desk PC in every two seconds.

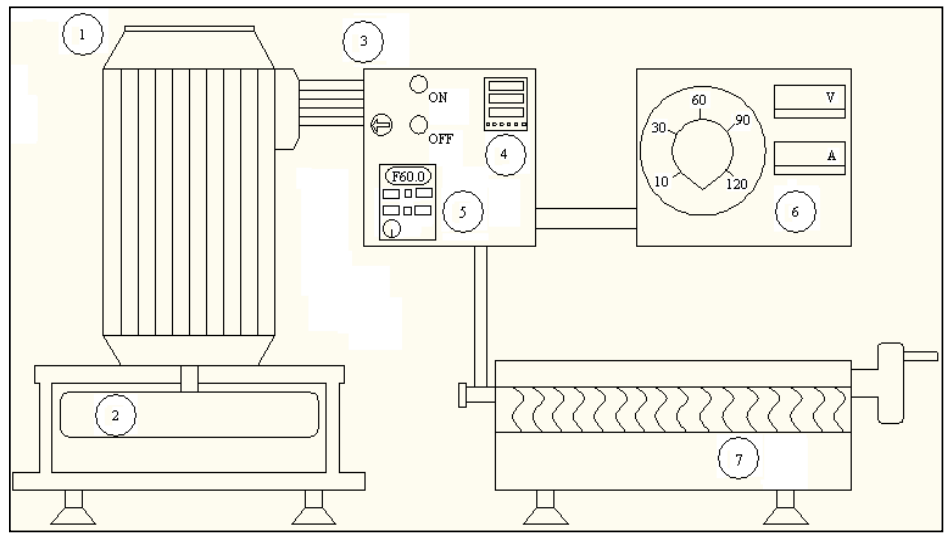

Figure 1: System Design

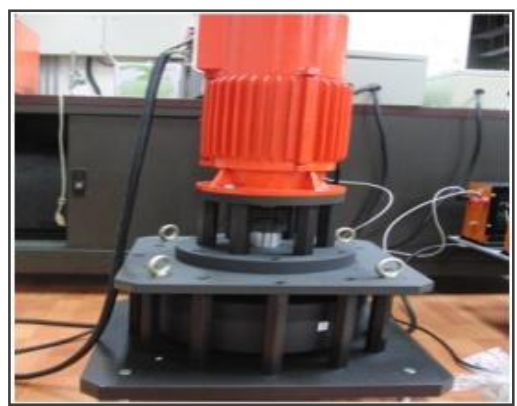

Figure 2: System setup

Table 1: System parameters

\begin{tabular}{|c|c|c|c|}
\hline Motor rated voltage & $220 \mathrm{~V}$ & Flywheel weight & $60 \mathrm{~kg}$ \\
\hline Motor rated current & $1.9 \mathrm{~A}$ & Flywheel Material & Iron \\
\hline Motor horsepower & $2 \mathrm{HP}$ & Flywheel radius & $\mathbf{0 . 1 5 m}$ \\
\hline
\end{tabular}

\section{FUNDAMENTAL KNOWLEDGE REQUIREMENT}

Before participating to this study, some of the fundamental principles in physics and calculation skills to ordinary linear equations are required for students as Newton's law of motion, feedback control, and Laplace transformation etc..

FE energy storage. 
According to the Newton's Second Law of Motion, the rotational kinetic energy $\mathrm{E}_{\mathrm{c}}(\mathrm{J})$ can be expressed as Eq. (1) as the flywheel is driven.

$$
\mathrm{E}_{C}=\frac{1}{2} I \omega^{2}
$$

where, $/\left(\mathrm{kg}-\mathrm{m}^{2}\right)$ is the rotational inertia of flywheel (or moment of inertia), and $\omega(\mathrm{rad} / \mathrm{s})$ is the rotational speed of flywheel.

The moment of inertia depends on the object's mass distribution. Its definitions is the second moment of mass with respect to distance $r$ from an axis integrating over the entire mass $m$. When the object is a solid disc-type ingot with weight $M(\mathrm{~kg})$ and the radius is $R(m)$, moment of inertia / can be expressed in Eq.(2).

$$
I=\int_{m} r d m=\frac{1}{2} M R^{2}
$$

By substituting Eq. (2) to Eq.(1), the energy storaged in the rotating object can be found as;

$$
\mathrm{E}_{C}=\frac{1}{4} M R^{2} \omega^{2}
$$

For the rotational inertia can be calculated and changed via adding or descreasing the number of disc-type ingots, makes the experimental works become simplified. That is, participators can only measure and then record the variation of rotational speed which subject to the input frequencies of driving motor.

\section{Parameter identification.}

In order to find the model of an unknown system presenting in the type of a continuous function, first, a second-order transfer function with three unknown parameters was given for system identification. Next, through identifying the parameters of a transfer function which characterizes an unknown system, the model was able to accurately obtain. Finally, using the well-identified transfer function via the calculation of inverse Laplace transform, a differential equation with time variance as well as a continuous function representing the unknown system could be obtained.

In this study, A simple method for fitting the parameters of a transfer function with overdamped responses (Chen, 1989) was used. The approximate model of a controlled object is given as a second-order transfer function with time-delay term as

$$
G(S)=\frac{C \infty}{\tau^{2} S^{2}+2 \zeta \tau S+1} e^{-d s}
$$


where, the parameters, including $C \infty, \zeta, \tau$ and time delay constant $d$, are unknown. For finding model, initially the system should be set in the open-loop state, then inputting a unit step signal to obtain the step response of the system as shown in Fig. 3.

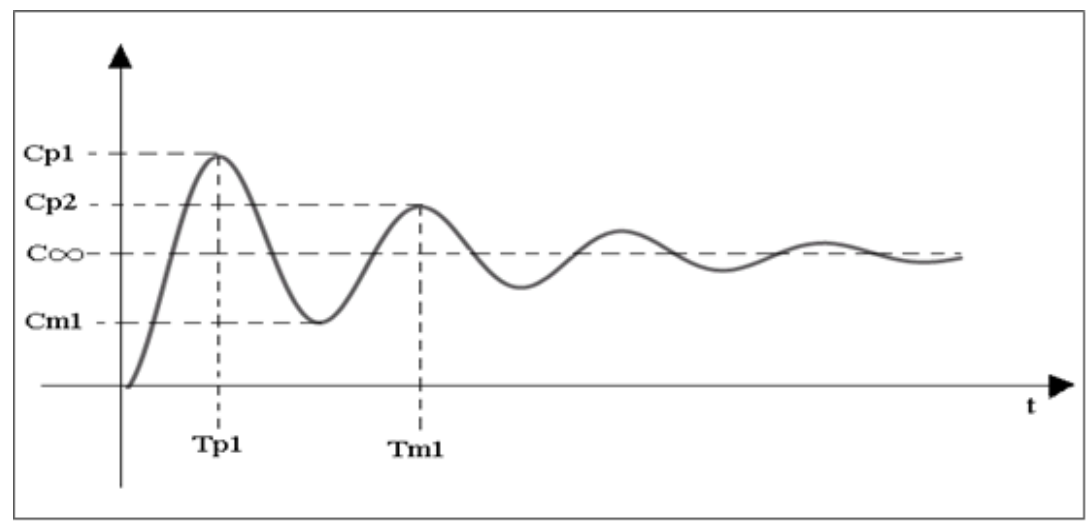

Figure 3: Step response of a system

The equations describing the relation of the values in Fig. 3 and the unknown parameters including $C \infty, \zeta, \tau$ and time delay constant $d$ are expressed as follows.

$$
\begin{gathered}
C \infty=\frac{C p 1 C p 2-C m 1^{2}}{C p 1+C p 2-2 C m 1} \\
\zeta=\frac{-\ln (H)}{\sqrt{\pi^{2}+\ln ^{2}(H)}} \\
H=\frac{1}{3}\left[\frac{C p 1-C \infty}{C \infty}+\frac{C \infty-C m 1}{C p 1-C \infty}+\frac{C p 2-C \infty}{C \infty-C m 1}\right] \\
\tau=\frac{(T m 1-T p 1) \sqrt{1-\zeta^{2}}}{\pi} \\
d=2 T p 1-T m 1
\end{gathered}
$$

A second-order transfer function with four unknown parameters was used to represent the unknown system for modeling as;

$$
T_{i}(s)=\sum_{i=1}^{n} \frac{a_{i}}{b_{i} s^{2}+c_{i} s+1} e^{-d_{i} s}
$$

By observing the data of step response found by experiment and substituting them into Eq.(5)-Eq.(9), the initial values of $a_{i}, b_{i}, c_{i}$, and $d_{i}$ could be obtained. 


\section{ANFIS model.}

Fig. 4 illustrates the parameter approaching mechanism of transfer function via ANFIS (Huang and Chang, 2011). The objective function is to minimize the difference $e(\mathrm{t})$ between the real system response $T_{\mathrm{r}}(\mathrm{t})$ and the calculated result Ti(t). Here, a threshold value $\eta$ and an error tolerance $\varepsilon$ are set for system identification as;

if $|e(t)-e(t-1)|>\varepsilon$, then continue calculation.

if $|e(t)-e(t-1)| \leq \varepsilon$ but $\left|g_{r}(t)-g(t)\right|>\eta$, then $i=i+1$ continue identification.

if $|e(t)-e(t-1)| \leq \varepsilon$ and $\left|g_{r}(t)-g(t)\right| \leq \eta$, then end identification.

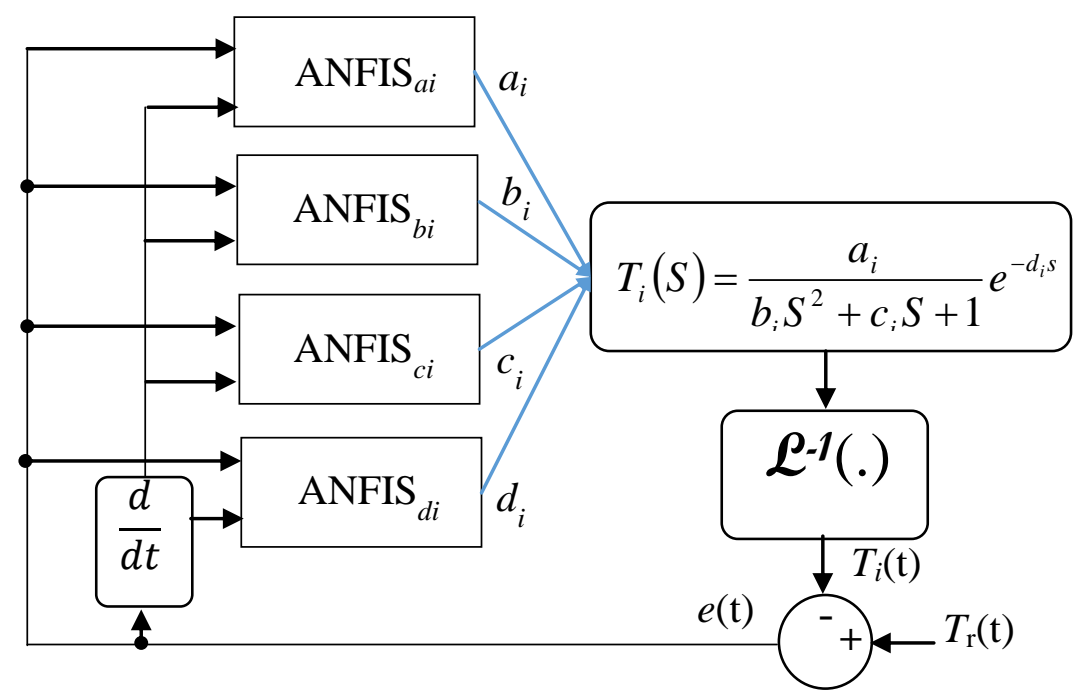

Figure 4: Parameter identification via ANFIS

\section{CASE STUDIES}

Due to learning the operational skills of experiment in engineering is one of the most important motives in this study. To let students familiar with the whole operational flow of the part in physical experiment, an energy-charging-and-discharging experiment via the designed EP system was taken place. The data of input and output energies were obtained via the experiments on the EP system subjected to the input variables as frequency variations, which were equivalently separated into nine parts in the range of 20 to $60 \mathrm{~Hz}$. On the other hand, the performance of discharging experiment was achieved via tuning the magnetizing currents at the values of $25 \%, 50 \%, 75 \%$ and $100 \%$ of satisfactions. According to Eq.(3) to Eq.(1), the input and output energies of EF can be found by using the measured EP rotational speeds which were recorded via a PC in every two seconds. 


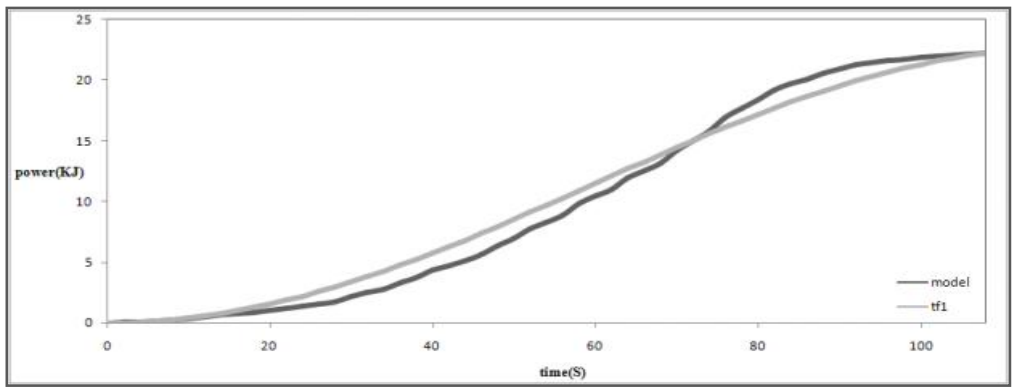

(a) by using single transfer function

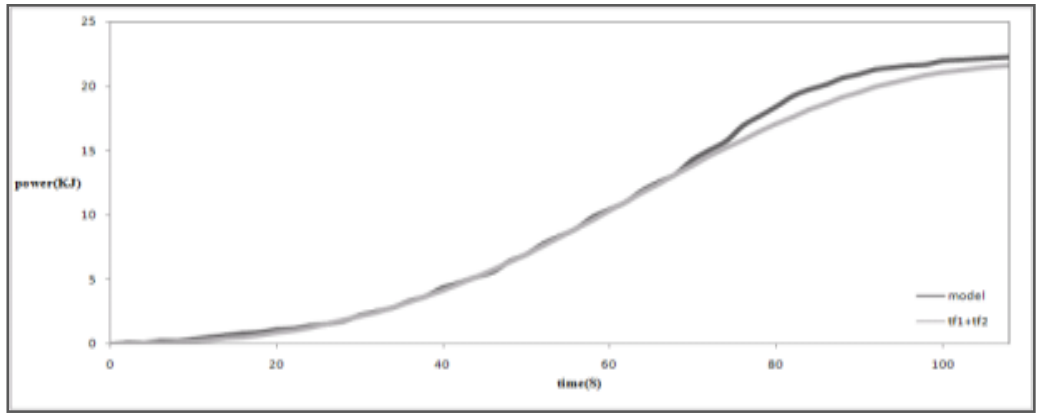

(b) by using two transfer functions

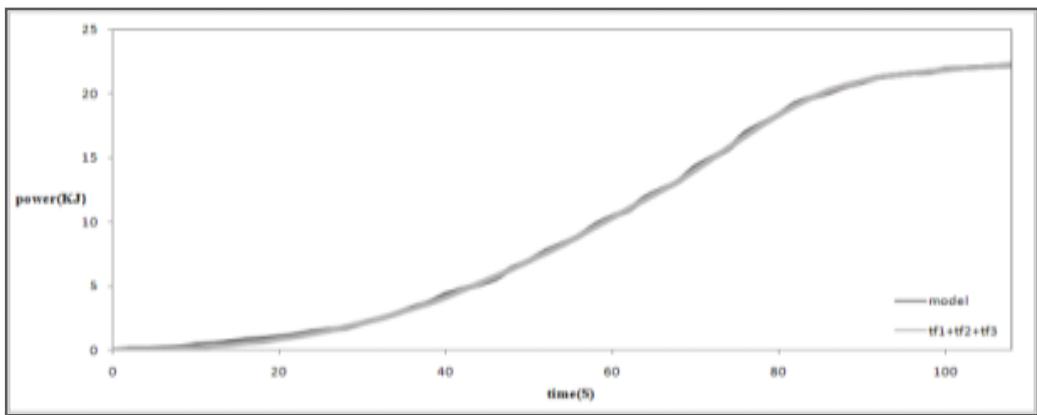

(c) by using three transfer functions

\section{Figure 5: Approaching performance}

Through ANFIS model, Fig. 5 shows the approaching performance by using single transfer function. Obviously, the error between model and real data is still large. According to the approaching mechanism in Fig. 4, for the error $e(\mathrm{t})$ is larger than the threshold value $\eta$, then $i=i+1$ another transfer function was added for modeling. Fig. 5(b) shows the approaching performance by using two transfer functions. To compare with Fig. 5(a), the error $e(\mathrm{t})$ in lower part of the curves was well-compensated by the added transfer function. However, for the error in the upper part of the curves was still existing, then, according to the 
approaching mechanism in Fig. 4, again, another transfer function was added. Fig. 5(c) shows the approaching performance by using three transfer functions. Obviously, the errors mentioned in above were completely compensated by using three transfer functions.

\section{CONCLUSION}

In this study, a completed trainning process for EEE was designed and proposed. Here, the potential students who are going to be the instructors in laboratories or engineering can learn how to design a studing flow for undergraduate students via physical experiments. In this paper, the flywheel stiorage system was setup and used to be a nonliner case for learning how to identify and then modeling by ANFIS, a model-free tool. It would be with much educational benefit on realizing what the importance is in data collection and analysis, phenomena of energy transformation and the applications of artifical intelligent technologies. In the near future, the evaluation of learning effectiness will be comfirmed through LDC (Literacy Design Collaborative) modules and questionnaire (http://ldc.org/).

\section{REFERENCES}

Beno J., Hebner R., and Walls A., (2002). Flywheel batteries come around again, IEEE Spectrum., 39, 46-51.

Chen, C.L., (1989). A simple method for on-line identification and controller tuning. AIChE journal, 35(12): p. 2037-2039.

Doerr H. M. and Lyn D., (2003). A modeling perspective on students' mathematical reasoning about data, English Journal for Research in Mathematics Education, 34(2), p. 110-136.

Dym CL , Agogino AM , Eris O , Frey DD, (2005). Engineering design thinking, teaching, and learning, Journal of Engineering Education, 94(1), p.103-120.

Ho, W.H., et al., (2009). Adaptive network-based fuzzy inference system for prediction of surface roughness in end milling process using hybrid Taguchi-genetic learning algorithm. Expert Systems with Applications, 36(2): p. 3216-3222.

Huang, C.N. and Chang,C.C., (2011). Optimal-Parameter Determination by Inverse Model based on MANFIS: The Case of Injection Molding for PBGA, IEEE Transactions on Control Systems Technology, 19(6), pp. 1596-1603.

Jang, J.S.R., 1993, ANFIS: adaptive-network-based fuzzy inference system. Systems, IEEE Transactions on Man and Cybernetics, 23(3): p. 665-685. 